\title{
Differential microRNA expression in the prefrontal cortex of mouse offspring induced by glyphosate exposure during pregnancy and lactation
}

\author{
HUA JI $^{1}$, LINHAO XU ${ }^{1}$, ZHENG WANG ${ }^{1}$, XINLI FAN ${ }^{1}$ and LIHUI WU ${ }^{2}$
}

Departments of ${ }^{1}$ Basic Medicine and ${ }^{2}$ Clinical Medicine, Hangzhou Medical College, Hangzhou, Zhejiang 310053, P.R. China

Received July 20, 2017; Accepted October 26, 2017

DOI: $10.3892 / \mathrm{etm} .2017 .5669$

\begin{abstract}
Glyphosate is the active ingredient in numerous herbicide formulations. The role of glyphosate in neurotoxicity has been reported in human and animal models. However, the detailed mechanism of the role of glyphosate in neuronal development remains unknown. Recently, several studies have reported evidence linking neurodevelopmental disorders (NDDs) with gestational glyphosate exposure. The current group previously identified microRNAs (miRNAs) that are associated with the etiology of NDDs, but their expression levels in the developing brain following glyphosate exposure have not been characterized. In the present study, miRNA expression patterns were evaluated in the prefrontal cortex (PFC) of 28 postnatal day mouse offspring following glyphosate exposure during pregnancy and lactation. An miRNA microarray detected 55 upregulated and 19 downregulated miRNAs in the PFC of mouse offspring, and 20 selected deregulated miRNAs were further evaluated by quantitative polymerase chain reaction (PCR). A total of 11 targets of these selected deregulated miRNAs were analyzed using bioinformatics. Gene Ontology (GO) terms associated with the relevant miRNAs included neurogenesis (GO:0050769), neuron differentiation (GO:0030182) and brain development (GO:0007420). The genes Cdknla, Numbl, Notchl, Fosll and Lefl are involved in the Wnt and Notch signaling pathways, which are closely associated with neural development. PCR arrays for the mouse Wnt and Notch signaling pathways were used to validate the effects of glyphosate on the expression pattern of genes involved in the Wnt and Notch pathways. Nr4a2 and Wnt7b were downregulated, while Dkkl, Dixdcl, Runx1, Shh, Lef-1 and Axin2 were upregulated in the PFC of mice offspring following glyphosate exposure during pregnancy and lactation. These results indicated abnormalities of
\end{abstract}

Correspondence to: Mr. Hua Ji, Department of Basic Medicine, Hangzhou Medical College, 481 Binwen Road, Hangzhou, Zhejiang 310053, P.R. China

E-mail: jihua@hmc.edu.cn

Key words: glyphosate, microRNA, prefrontal cortex, mice offspring, bioinformatic analysis the Wnt/ $\beta$-catenin and Notch pathways. These findings may be of particular interest for understanding the mechanism of glyphosate-induced neurotoxicity, as well as helping to clarify the association between glyphosate and NDDs.

\section{Introduction}

Glyphosate is a phosphonomethyl amino acid derivative that is the active ingredient in a number of herbicides (1). Glyphosate-based herbicides are the most highly utilized agrochemicals in the world, particularly on genetically modified plants (2). Extensive evidence demonstrates that the large scale application of glyphosate causes high amounts of residue in water and soil (3). This residue was not previously considered to pose any risks to human health (4). However, recently, glyphosate has been demonstrated to induce embryo-toxic and neurotoxic effects in in vitro and in vivo studies $(5,6)$. The teratogenic effect of glyphosate on early morphogenesis in embryos raises concerns regarding the clinical phenomenon including, birth defects and behavior disorders, seen in children exposed to glyphosate in the countryside $(2,7)$. Therefore, the safety of glyphosate remains controversial.

In vitro studies have revealed that glyphosate can pass through the blood brain barrier and placental barrier (8). Evidence has also indicated that glyphosate causes widespread apoptotic neurodegeneration, as well as effects on neuronal development and axon growth (9). Results from the Childhood Autism Risks from Genetics and Environment study provide further evidence for an association between neurodevelopmental disorders (NDDs) and gestational organophosphate exposure, particularly glyphosate (10). However, the detailed mechanism of glyphosate neurotoxicity in the developing brain is not well understood. Similarly, while the exact etiology of NDDs remains unknown, novel studies have provided insight into the possible role of environmental and epigenetic factors in the etiology of NDDs $(11,12)$.

MicroRNAs (miRNAs) are small, non-coding RNAs that are recognized as endogenous regulators of post-transcriptional gene expression (13). They are involved in numerous biological processes, including the cell cycle, cell proliferation, the cellular response to stress (14) and the regulation of gene expression (15). Increasing evidence indicates that miRNAs are dysregulated in numerous diseases, including NDDs such as attention deficit hyperactivity disorder (ADHD) and autism 
spectrum disorder (ASD). Multiple circulating miRNAs have been demonstrated to be differentially expressed in child patients with a range of NDDs compared with healthy children (16). Development of the prefrontal cortex (PFC), which is the brain region most affected in ADHD (17), may be associated with regulation of gene expression by miRNAs, which are numerous in the brain (18). A previous study by the current group indicated that miRNA let-7d was elevated in the serum of ADHD subjects (19), as well as in the PFC of spontaneously hypertensive rats, which were used as an ADHD model (20).

Considering the pivotal role of miRNAs in the regulation of gene expression and neurodevelopment dysfunction, a miRNA microarray method was used in the present study to investigate miRNA expression changes in the PFC of mouse offspring following glyphosate exposure during pregnancy and lactation. Furthermore, certain significantly altered miRNAs associated with brain development were selected to perform bioinformatics analysis. This included target gene prediction, Gene Ontology (GO) term enrichment and Kyoto Encyclopedia of Genes and Genomes (KEGG) pathway analysis. The aim of the present study was to reveal the potential function of these miRNAs in the PFC of mice offspring, as well as the mechanism of glyphosate neurotoxicity in the developing brain.

\section{Materials and methods}

Sample preparation. All procedures were approved by the Institutional Animal Care and Use Committee of Hangzhou Medical College (Hangzhou, China) and conformed to the guidelines for ethical treatment of animals. Experiments required collecting RNA samples from PFCs isolated from postnatal day (PND) 28 male mice, with the day of birth considered as PND 0. The pesticide used in the present study was a commercial formulation marketed in China as Roundup ${ }^{\circledR}$ (Monsanto Company, St. Louis, MO, USA), containing $48 \mathrm{~g}$ glyphosate isopropylamine salt per $100 \mathrm{~cm}^{3}$ of product (equivalent to $35.6 \% \mathrm{w} / \mathrm{v}$ of Glyphosate acid).

All experiments were performed in accordance with the China Council of Animal Care and approved by the Hangzhou Medical College Animal Care Committee. A total of 18 pregnant ICR mice (age, 9-11 weeks; weight 40-50 g; Shanghai Laboratory Animal Center, Chinese Academy of Sciences, Shanghai, China) were randomly divided into two groups, with each group consisting of 8 pregnant mice. All mice were given free access to food and water and were maintained in a $12 \mathrm{~h} \mathrm{light/dark} \mathrm{cycle} \mathrm{in} \mathrm{a} \mathrm{temperature-controlled} \mathrm{breeding}$ room $\left(21^{\circ} \mathrm{C}\right)$ with $45-60 \%$ humidity and $<66 \pm 2 \mathrm{~dB}$ room noise level. Each group was used for the miRNA microarray assay and the polymerase chain reaction (PCR) array. In the control group, pregnant mice were provided with purified water. In the glyphosate-treated group, pregnant mice were provided with drinking water containing $0.38 \%$ glyphosate $\left(1 \%\right.$ Roundup $\left.{ }^{\circledR}\right)$ during pregnancy and lactation, equivalent to $50 \mathrm{mg}$ of glyphosate $/ \mathrm{kg} /$ day. This dose corresponded with $1 / 20$ th of the glyphosate no-observed-adverse-effect level, as described previously (4). The mothers received treatment from embryonic day (E) 14 to PND 7 and were then provided with normal drinking water. The offspring received it indirectly via pregnancy and lactation and weaning occurred on PND 21, they were then provided with normal drinking water. A total of 8 offspring (4 females and 4 males) from each group were sacrificed on PND 28, the brains were quickly removed, and the PFC was isolated on an ice pad.

Total RNA extraction. Total RNA was isolated using TRIzol (Invitrogen; Thermo Fisher Scientific, Inc., Waltham, MA, USA) and purified with an RNeasy Mini kit (Qiagen GmbH, Hilden, Germany), according to the manufacturer'sprotocol. The concentration of RNA was determined by measuring the absorbance at $260 \mathrm{~nm}$ (A260) by a NanoDrop spectrophotometer (ND-1000, NanoDrop Technologies; Thermo Fisher Scientific, Inc.), the value of A260/A280 provided an estimate of the purity of RNA. When the RNA samples complied with an A260/A280 ratio of 1.8-2.0, the RNA analysis could proceed and RNA integrity was determined by $1.2 \%$ agarose gel electrophoresis.

miRNA microarray hybridization. Profiling of miRNA expression was performed using miRCURY LNA ${ }^{\mathrm{TM}}$ microRNA Array v19.0, 7th generation, hsa, mmu \& rno (Exiqon, Inc., Woburn, MA, USA). The microarray contained 3,100 capture probes, which cover all human, mouse and rat miRNAs annotated in the miRBase (release 19) (mirbase.org/). The total isolated RNA was taken from pooled samples of each group. They were then labeled using the miRCURY ${ }^{\mathrm{TM}}$ Power Labeling kit (Exiqon, Inc.) and hybridized to miRCURY $\mathrm{LNA}^{\mathrm{TM}}$ miRNAs Array v19.0, according to the manufacturer's protocol. Hybridization image scanning was performed using the Axon GenePix 4000B microarray scanner (Molecular Devices, LLC, Sunnyvale, CA, USA).

miRNA microarray analysis. Scanned images were imported into GenePix Pro 6.0 software (Molecular Devices, LLC) for grid alignment and data extraction. Replicated miRNAs were averaged, and miRNAs with intensities $\geq 30$ in all samples were selected for calculating a normalization factor. Expressed data were normalized using the median normalization. Then, significant, differentially expressed miRNAs between the two groups were identified using fold change and P-values. Differentially expressed miRNAs between two samples were filtered through fold change. The value of the miRNAs was the foregound intensity of each probe. The normalized ratio of the miRNA's foreground and background and other data were deposited in the NCBI Gene Expression Omnibus and are accessible online (accession no. GSE100079; www.ncbi. $\mathrm{nlm}$.nih.gov/geo/query/acc.cgi?acc=GSE100079). Filtering was performed to identify differentially expressed miRNAs with fold changes $\geq 2.0$ and P-values $\leq 0.05$.

Reverse transcription-quantitative PCR (RT-qPCR). Based on previous research $(21,22), 20$ miRNAs (mmu-miR-322-5p, mmu-miR-376b-5p, mmu-miR-592-5p, mmu-miR-142a-5p, mmu-miR-540-5p, mmu-miR-181a-5p, mmu-miR-183-3p, mmu-miR-470-5p, mmu-miR-19b-3p, mmu-let-7a-5p, mmu-miR-376a-5p, mmu-miR-381-3p, mmu-miR-3475-3p, mmu-miR-34b-5p, mmu-miR-320-3p, mmu-miR-484, mmu-miR-93-5p, mmu-miR-375-5p, mmu-miR-34c-5p, mmu-miR-3098-3p) were selected that are considered to be relevant to brain development. To validate the accuracy of the miRNA microarray data, the RNAs were polyadenylated 
through a poly (A) polymerase reaction using the $\mathrm{MystiCq}^{\circledR}$ microRNA cDNA Synthesis mix (Sigma-Aldrich; Merck, KgaA, Darmstadt, Germany) and then reverse transcribed into cDNA by ReadyScript ${ }^{\mathrm{TM}}$ reverse transcriptase and oligo-dT adapter primers (Sigma-Aldrich; Merck KGaA). Individual miRNAs were quantified using SYBR Green qPCR ReadyMix ${ }^{\mathrm{TM}}$. Reverse primers were $\mathrm{MystiCq}^{\circledR}$ Universal PCR Primer (Sigma-Aldrich; Merck KGaA) and the forward primers were the specific MystiCq miRNA qPCR assay primers, mmu-miR-34b-5p, 5'-AGGCAG TGTAATTAGCTGATTGT-3'; mmu-miR-322-5p, 5'-CAG CAGCAATTCATGTTTTGGA-3'; mmu-miR-376b-5p, 5'-GTGGATATTCCTTCTATGGTTA-3' purchased from Sigma-Aldrich (Merck KGaA) and Wcgene Biotechnology Corporation (Shanghai, China) synthetic primers as follows: mmu-let-7a-5p, 5'-TGAGGTAGTAGGTTGTATAGTT-3'; mmu-miR-19b-3p, 5'-TGTGCAAATCCATGCAAAACT GA-3'; mmu-miR-34c-5p, 5'-AGGCAGTGTAGTTAGCTG ATTGC-3'; mmu-miR-93-5p, 5'-CAAAGTGCTGTTCGT GCAGGTAG-3'; mmu-miR-142a-5p, 5'-CATAAAGTAGAA AGCACTACT-3'; mmu-miR-181a-5p, 5'-AACATTCAACGC TGTCGGTGAGT-3'; mmu-miR-183-3p, 5'-GTGAATTAC CGAAGGGCCATAA-3'; mmu-miR-320-3p, 5'-AAAAGC TGGGTTGAGAGGGCGA-3'; mmu-miR-375-5p, 5'-GCG ACGAGCCCCTCGCACAAAC-3'; mmu-miR-376a-5p, 5'-GGTAGATTCTCCTTCTATGAGT-3'; mmu-miR-381-3p, 5'-TATACA AGGGCA AGC TCTCTG T-3'; mmu-miR470-5p, 5'-TTC TTG GAC TGG CAC TGG TGA GT-3'; mmu-miR-484, 5'-TCAGGCTCAGTCCCCTCCCGAT-3'; mmu-miR-540-5p, 5'-CAAGGGTCACCCTCTGACTCT GT-3'; mmu-miR-592-5p, 5'-ATTGTGTCAATATGCGAT GATGT-3'; mmu-miR-3098-3p, 5'-TTCTGCTGCCTGCCT TTAGGA-3'; mmu-miR-3475-3p, 5'-TCTGGAGGCACAT GGTTTGAA-3'; U1, 5'-CTTACCTGGCAGGGGAGATA-3'. The protocol of miRNA RT-qPCR array analysis was as previously described (23) and as specified on the Wcgene website (wcgene.com). The mouse U1 small nuclear rna gene were used to normalize expression. The $2^{-\Delta \Delta \mathrm{Cq}}$ method (24) was used to determine differences in expression level between the glyphosate and the control group. $\mathrm{P}<0.05$ was considered to indicate a statistically significant difference. The miRNA RT-qPCR array experiments were conducted at Wcgene Biotechnology Corporation (Shanghai, China).

Target gene prediction and bioinformatics analysis. Based on existing research and the RT-qPCR results the potential function of 11 miRNAs was explored (mmu-miR-142a-5p, mmu-miR-181a-5p, mmu-miR-19b-3p, mmu-miR-322-5p, mmu-miR-470-5p, mmu-miR-540-5p, mmu-miR-320-3p, mmu-miR-324-5p, mmu-miR-34b-5p, mmu-miR-484, mmu-miR-93-5p), TargetScan (targetscan. org/vert_71/), miRanda (microrna.org/) and PicTar (pictar. org/) software was used to predict target mRNAs. Then, miRNA function was explored further using the GO database (geneontology.org/) as an analysis tool for target genes of the predicted miRNAs. The pathways of the miRNA targets were then explored using the KEGG functional annotation analysis (genome.jp/kegg/). The results indicated that a large number of the target genes were involved in the Wnt and Notch signaling pathways.
Wnt and Notch signaling pathway PCR array. On the basis of the KEGG functional annotation analysis, the mouse Wnt and Notch signaling pathway $\mathrm{RT}^{2}$ profiler $^{\mathrm{TM}}$ PCR array plates (Wcgene Biotechnology Corporation) were used, which contained 84 key genes involved in the Wnt pathway and 26 key genes involved in the Notch pathway. The reaction was performed according to the manufacturer's protocol. Real-time PCR was performed using SYBR-Green Master mix (Qiagen $\mathrm{GmbH}$ ) and processed in the GeneAmp 5700 Sequence Detection system (Applied Biosystems; Thermo Fisher Scientific). The data was exported to and analyzed by Wcgene Biotechnology Corporation.

Statistical analysis. SPSS version 16.0 (SPSS, Inc., Chicago, IL, USA) was used to perform statistical analyses. Data are presented as the mean \pm standard error of the mean. Aspin-Welch's t-test was applied to identify genes and miRNAs that demonstrated a significant differential expression upon exposure to glyphosate. $\mathrm{P}<0.05$ was considered to indicate a statistically significant difference.

\section{Results}

miRNA expression analysis. The results of the miRCURY LNA $^{\mathrm{TM}}$ miRNA microarray assay identified 74 miRNAs that were differentially expressed in the two groups with $\mathrm{P}<0.01$ and signal values $>500$. In the glyphosate group, 55 miRNAs were upregulated and 19 miRNAs were downregulated compared with the control group (Table I and Fig. 1).

Single validation of miRNA by $q P C R$. To confirm the accuracy of the miRNA microarray, 20 differentially expressed, representative miRNAs, which were considered relevant to brain development were selected based on previously published studies and used for qPCR, $(25,26)$. The results demonstated that the expression levels of 12 miRNAs, including miR-322-5p and miR-19b-3p, were upregulated in the glyphosate group. The expression levels of 7 miRNAs, including miR-34b-5p and miR-320-3p, were downregulated (Fig. 2). These results were in accordance with the miRNA microarray data.

miRNA predicted targets and relevant bioinformatic analysis. To better recognize the role of miRNA expression in biosynthesis, three software packages (TargetScan, PicTar and miRanda) were used for target prediction, GO enrichment analysis and KEGG annotation analysis. A total of 11 putative target genes from miRNAs in the two groups were identified (Table II). These genes are associated with neuronal development, suggesting that miRNAs are involved in regulating the pathological processes of glyphosate-induced neurotoxicity through the targeting of these genes.

These putative miRNA targets were used as inputs to perform the GO functional enrichment. The $26 \mathrm{GO}$ terms identified to be associated with neuronal development are shown in Table III, including neurogenesis (GO:0050769), neuron differentiation (GO:0030182) and brain development (GO:0007420). These results demonstrate that the target genes of miRNAs in mouse PFC may be involved in a wide variety of pathophysiological processes following glyphosate exposure. 
Table I. Differentially expressed miRNAs in the prefrontal cortex between the glyphosate and control groups analyzed by microarray at a signal value $>500$ and $\mathrm{P}<0.01$.

A, Upregulated miRNAs in the glyphosate group

\begin{tabular}{|c|c|c|c|}
\hline miRNA & $\begin{array}{l}\text { Control } \\
\text { group }\end{array}$ & $\begin{array}{l}\text { Glyphosate } \\
\text { group }\end{array}$ & $\begin{array}{l}\text { Fold- } \\
\text { change }\end{array}$ \\
\hline mmu-miR-711 & 68 & 82 & 2.0276 \\
\hline mmu-miR-27b-3p & 126.5 & 274.5 & 3.55381 \\
\hline mmu-miR-381-3p & 65.5 & 111 & 4.75526 \\
\hline mmu-miR-425-5p & 69 & 89.5 & 2.20282 \\
\hline mmu-miR-872-5p & 79.5 & 99 & 2.13158 \\
\hline mmu-miR-592-5p & 62.5 & 96 & 10.3658 \\
\hline mmu-miR-434-3p & 891.5 & 1,777 & 2.12625 \\
\hline mmu-miR-181a-5p & 223 & 985.5 & 5.69772 \\
\hline mmu-miR-130a-3p & 354 & 772.5 & 2.49714 \\
\hline mmu-miR-30a-5p & 457 & $1,319.5$ & 3.24703 \\
\hline mmu-miR-27a-3p & 80.5 & 100 & 2.45888 \\
\hline mmu-miR-374b-5p & 65 & 87 & 2.58045 \\
\hline mmu-miR-181c-5p & 85.5 & 170 & 4.27632 \\
\hline mmu-miR-136-5p & 466 & $1,436.5$ & 3.49801 \\
\hline mmu-miR-29b-3p & 736 & 2512 & 3.70614 \\
\hline mmu-miR-204-3p & 76 & 98.5 & 2.07237 \\
\hline mmu-miR-7b-5p & 70.5 & 94.5 & 3.6098 \\
\hline mmu-miR-128-3p & 2263 & 5,590 & 2.56915 \\
\hline mmu-miR-1958 & 78.5 & 100 & 2.14266 \\
\hline mmu-miR-466m-5p & 64 & 73.5 & 2.2359 \\
\hline mmu-miR-126a-5p & 158.5 & 466 & 4.10526 \\
\hline mmu-miR-98-5p & 319 & 1,104 & 4.10139 \\
\hline mmu-miR-540-5p & 64 & 94.5 & 6.7352 \\
\hline mmu-miR-376b-5p & 58.5 & 167 & 16.8316 \\
\hline mmu-miR-9-5p & $6,291.5$ & 12,794 & 2.09456 \\
\hline mmu-miR-129-2-3p & 292.5 & 694.5 & 2.66427 \\
\hline mmu-miR-344b-3p & 83 & 150 & 2.93836 \\
\hline mmu-miR-410-3p & 166 & 364.5 & 2.93628 \\
\hline mmu-miR-470-5p & 64.5 & 72 & 5.21053 \\
\hline mmu-miR-204-5p & 81 & 113 & 2.58348 \\
\hline mmu-miR-219a-5p & 290 & 741.5 & 2.97483 \\
\hline mmu-miR-29c-5p & 68.5 & 103 & 3.48947 \\
\hline mmu-miR-19b-3p & 86 & 220 & 5.00505 \\
\hline mmu-miR-183-3p & 62 & 78 & 5.57143 \\
\hline mmu-miR-677-5p & 77 & 150 & 3.72895 \\
\hline mmu-miR-19a-3p & 128.5 & 233.5 & 2.6063 \\
\hline mmu-miR-1971 & 78 & 110 & 2.82237 \\
\hline mmu-miR-142a-3p & 95 & 199.5 & 4.26947 \\
\hline mmu-miR-148a-3p & 66 & 107.5 & 3.90662 \\
\hline mmu-miR-142a-5p & 59.5 & 99.5 & 9.88995 \\
\hline mmu-miR-383-5p & 76.5 & 101.5 & 3.37218 \\
\hline mmu-miR-693-5p & 64 & 84 & 2.25219 \\
\hline mmu-let-7a-5p & 171.5 & 590.5 & 4.88616 \\
\hline mmu-miR-21a-5p & 173 & 396 & 3.18842 \\
\hline mmu-miR-488-3p & 67 & 95 & 3.68058 \\
\hline mmu-miR-181b-5p & 92 & 177.5 & 3.88215 \\
\hline mmu-miR-145a-5p & 73 & 98.5 & 3.3676 \\
\hline
\end{tabular}

Table I. Continued.

A, Upregulated miRNAs in the glyphosate group

\begin{tabular}{lccc}
\hline miRNA & $\begin{array}{c}\text { Control } \\
\text { group }\end{array}$ & $\begin{array}{c}\text { Glyphosate } \\
\text { group }\end{array}$ & $\begin{array}{c}\text { Fold- } \\
\text { change }\end{array}$ \\
\hline mmu-miR-376a-5p & 57.5 & 78 & 4.81579 \\
mmu-miR-691 & 962 & $2,353.5$ & 2.62274 \\
mmu-miR-3068-3p & 232 & 413 & 2.14251 \\
mmu-miR-322-5p & 67.5 & 159.5 & 23.8105 \\
mmu-miR-30b-3p & 101 & 124 & 2.22819 \\
mmu-miR-365-3p & 132.5 & 264 & 3.29501 \\
mmu-miR-28a-5p & 75.5 & 106 & 3.66541 \\
mmu-miR-380-3p & 89 & 116.5 & 3.35885 \\
\hline
\end{tabular}

$\mathrm{B}$, Downregulated miRNAs in the glyphosate group

\begin{tabular}{lccc}
\hline miRNA & $\begin{array}{c}\text { Control } \\
\text { group }\end{array}$ & $\begin{array}{c}\text { Glyphosate } \\
\text { group }\end{array}$ & $\begin{array}{c}\text { Fold- } \\
\text { change }\end{array}$ \\
\hline mmu-miR-744-5p & 239 & 131.5 & 0.47581 \\
mmu-miR-34c-5p & 176.5 & 89.5 & 0.3677 \\
mmu-miR-486a-5p & 85.5 & 57 & 0.41863 \\
mmu-miR-132-5p & $1,478.5$ & 698.5 & 0.47413 \\
mmu-miR-500-3p & 90.5 & 62 & 0.49263 \\
mmu-miR-484 & 80 & 53 & 0.33676 \\
mmu-miR-467b-5p & 86 & 57.5 & 0.40789 \\
mmu-miR-3098-3p & 364.5 & 166 & 0.40067 \\
mmu-miR-342-3p & 187 & 100 & 0.47039 \\
mmu-miR-34b-5p & 240.5 & 86 & 0.26139 \\
mmu-miR-3072-3p & 194 & 113 & 0.49684 \\
mmu-miR-320-3p & 216.5 & 93.5 & 0.33023 \\
mmu-miR-195a-5p & $1,096.5$ & 487.5 & 0.43865 \\
mmu-miR-375-5p & 86 & 57 & 0.36154 \\
mmu-miR-133b-3p & 111 & 71 & 0.49221 \\
mmu-miR-93-5p & 170 & 84 & 0.34644 \\
mmu-miR-1839-3p & 379 & 193 & 0.48888 \\
mmu-miR-3475-3p & 96 & 48 & 0.08435 \\
mmu-miR-324-5p & 155 & 79.5 & 0.40297 \\
\hline man & & & \\
\hline
\end{tabular}

miRNA, microRNA.

KEGG functional annotation analysis. To evaluate the biological pathways involved in glyphosate-induced neurotoxicity, KEGG pathway annotation of the miRNA targets (Table IV) was performed. KEGG pathway analysis revealed certain biological processes that may be involved in glyphosate-induced neurotoxicity, and provided useful insights for further investigation of the role of targeted miRNAs in the neurotoxic effects of glyphosate on the developing brain.

The pathway that was most enriched was Ras, followed by mitogen-activated protein kinase and the cell cycle. Thus, KEGG analysis revealed certain biological processes that may be involved in glyphosate-induced neurotoxicity. In addition, it 


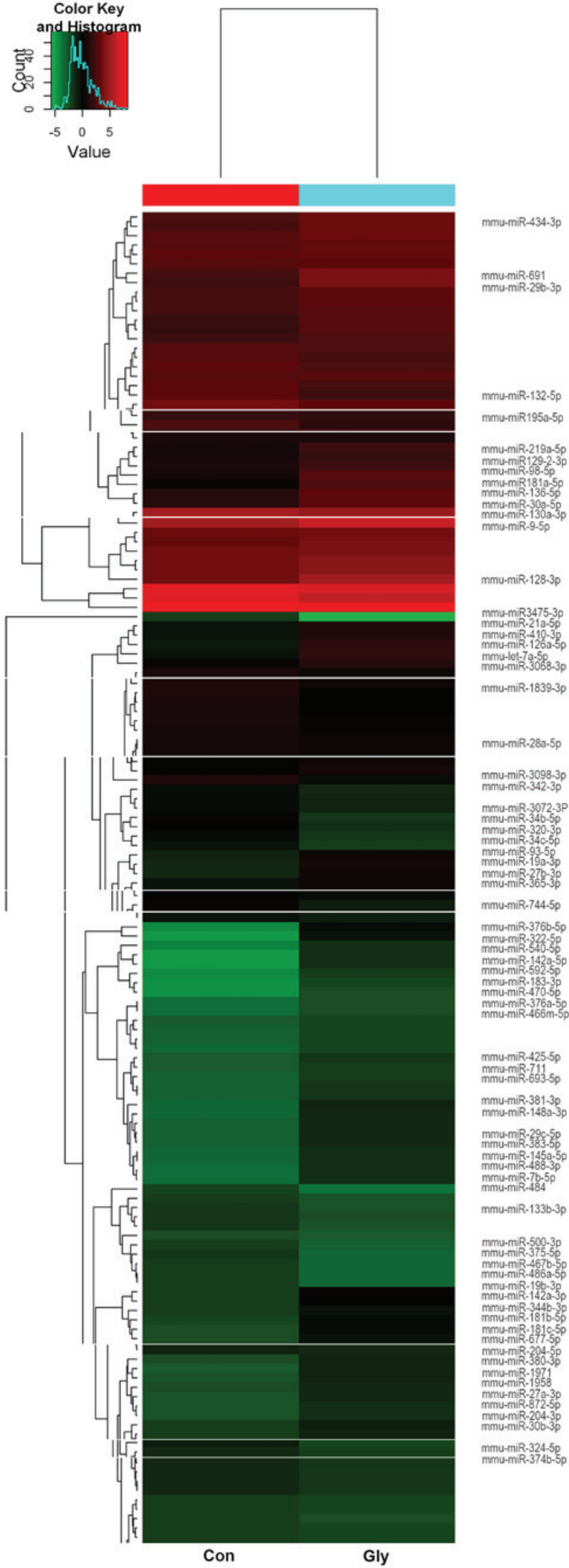

Figure 1. Heat map for the analysis of differentially-expressed miRNA. Each column represents a sample; each row represents an miRNA. Red color indicates upregulated miRNAs; green color indicates downregulated miRNAs in the glyphosate group. The heat map for $74 / 569$ detected miRNAs $(13.01 \%)$ that were differentially expressed, with $\mathrm{P}<0.01$ and signal value $>500$, is depicted. Con, control group; Gly, glyphosate-treated group; miRNA, microRNA. was identified that the genes Cdknla, Numbl, Notchl and Lefl were involved in the Wnt and Notch signaling pathways. The Wnt signaling pathway serves a key role in cell cycle progression and differentiation during dopaminergic neurogenesis in the midbrain, which is thought to be associated with normal motor behavior (27). Notch signaling also appears to regulate dopaminergic neuronal development (28). Therefore, the two pathways may potentially contribute to neurodegenerative diseases and NDDs.

To validate the effects of the Wnt and Notch signaling pathway, the mouse Wnt and Notch signaling pathway $\mathrm{RT}^{2}$ profiler $^{\mathrm{TM}}$ PCR array was used in the present study. Fig. 3 presents the differential expression profile of the Wnt and Notch signaling pathways in the two groups. Ten transcripts, including Nr4a2 and $W n t 7 b$, were downregulated by glyphosate exposure, while 25 transcripts, including Dkk1, Dixdcl, Runx1, Shh, Lef1 and Axin2, were upregulated by glyphosate exposure (Fig. 3).

\section{Discussion}

Glyphosate neurotoxicity is known to be associated with glutamate excitotoxicity and oxidative stress (29). Acute glyphosate exposure in adult rats causes behavioral changes as well as alterations in dopaminergic markers (1). Previous epidemiological studies have identified that organophosphate pesticide exposure may have deleterious effects on neurodevelopment, including an increased risk of ADHD in Taiwanese children (30) and young Mexican-Americans (31). Shelton et al (10) also reported evidence linking NDDs with gestational organophosphate exposure, particularly with glyphosate. Preliminary experiments by the current authors indicated that glyphosate induces neural behavior abnormalities in mice offspring at PND 28 and PND 42 (data not shown). However, the mechanism for these abnormalities is not clear.

Growing evidence indicates that the pathogenesis of NDDs is linked to the interaction between genetics and the environment (32). It has also been demonstrated that exposure to environmental risk factors at an early age could induce NDDs, a process mediated by epigenetics (33). Epigenetics refers to heritable changes in gene function that do not change the nucleotide sequence or alter the gene products' function, but do affect the gene's spatiotemporal expression (34). The genome is known to be shaped throughout the life cycle by environmental factors (35).

Recently, miRNAs have been recognized as critical regulators of neuronal activity and function (36). They can also silence genes by translational repression or mRNA degradation (37), and dramatically alter certain downstream signaling pathways (38). A previous study by the current group also identified that several miRNAs, including miRNA let-7d, were dysregulated in ADHD subjects (19) and in a rat model of ADHD (20).

Based on these previous findings, it was hypothesized that miRNA expression is involved in glyphosate neurotoxicity. To test this, miRNA expression profiles in the PFC of mouse offspring exposed to glyphosate during pregnancy and lactation were analyzed using the miRNA microarray method. In the present study, $0.38 \%$ glyphosate was administered (half the dose regularly used), since a similar dose was used in a 
Table II. Predicted miRNA target genes associated with neuronal or brain development.

\begin{tabular}{lrl}
\hline miRNA & Count & \multicolumn{1}{c}{ Target genes } \\
\hline mmu-miR-142a-5p & 4 & Bnip2, Mnat1, Otx2, Setd2 \\
mmu-miR-181a-5p & 11 & Ddit4, Dock7, Fos, Grm5, Inpp5e, Phox2b, Plc12, Sema4c, Six2, Slc9a6, Tgif2 \\
mmu-miR-19b-3p & 23 & Cntfr, Dlc1, Fkbp1b, Fos11, Kcnc4, Kif3a, Neurod1, Palb2, Pla2g10, Ppara, Ptprg, \\
& & Raf1, Pon2, Nr3c2, Rap1a, Rfx4, Scn1b, Slc9a6, Sphk2, Tgif1, Tnfrsf12a, Usp33, \\
mmu-miR-322-5p & 17 & Adrb2, Atp7a, Atxn2, Cnih2, Dll1, Epha7, Grm7, Katnb1, Kif1b, Lrp6, Omg, Ptprm, \\
& & Raf1, Rnf10, Sncg, Stxbp3a, Tgif2 \\
mmu-miR-470-5p & 3 & Bcl11a, Is11, Tgif1 \\
mmu-miR-540-5p & 2 & Efna4, Ncoa1 \\
mmu-miR-320-3p & 9 & Adam10, Kcnip4, Mapk8ip3, Myo10, Pik3ca, Plk3, Prkg1, Ulk1, Vim \\
mmu-miR-324-5p & 3 & Pbx1, Slitrk4, Unc5c \\
mmu-miR-34b-5p & 10 & Abr, Chl1, Cntnap1, Crhr1, Foxg1, Jag1, Lef1, Nrn1, Numbl, Notch1 \\
mmu-miR-484 & 6 & Crtc2, Dpys12, Kcnj11, Grik4, Sirt2, Strn \\
mmu-miR-93-5p & 17 & Arhgef7, Cdkn1a, E2f1, Epha5, Foxb1, Kif5a, Lhx8, Map3k5, Myh14, Neurog2, Ski, \\
& & Sorl1, Tnfrsf21, Vegfa, Vldlr, Wee1, Wfs1 \\
\hline
\end{tabular}

miRNA, microRNA.

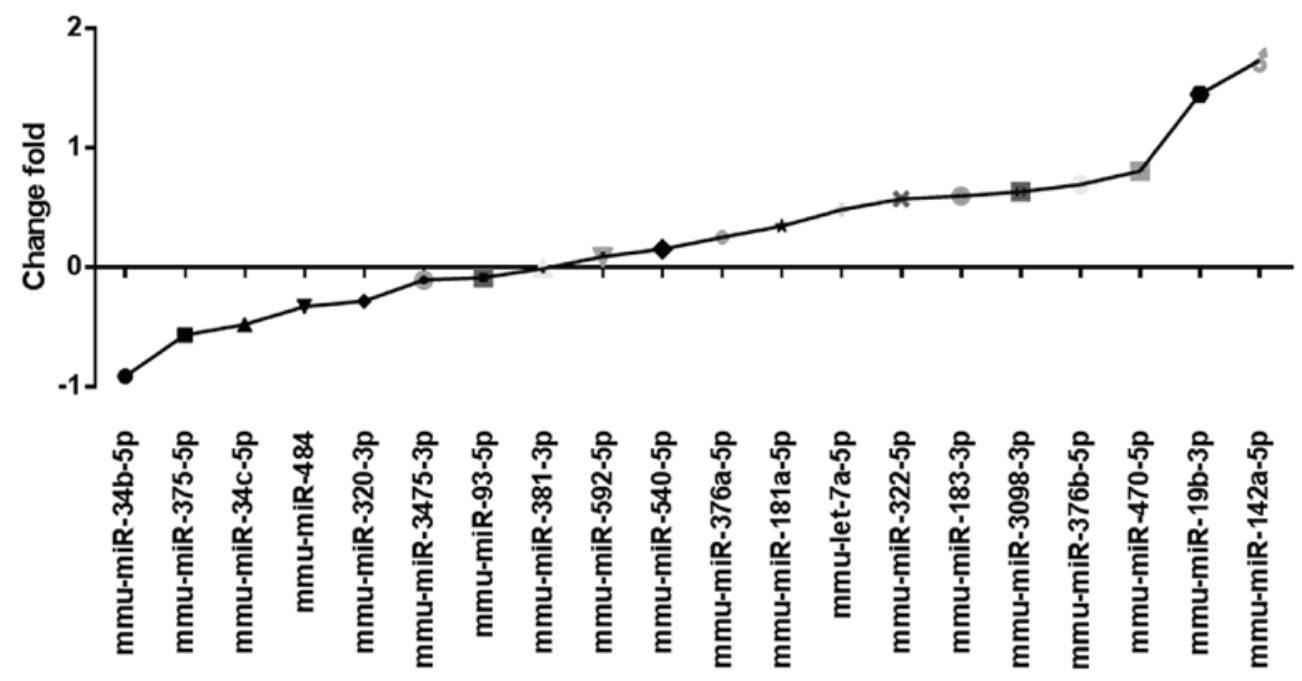

Figure 2. miRNA expression in the prefrontal cortex. A representative sample of 20 differentially-expressed miRNAs were selected and used for quantitative polymerase chain reaction. miRNA, microRNA.

previous study (5). The miRNA microarray is an efficient technique to analyze alternated miRNA expression profiles. The results indicated 53 differentially expressed miRNAs, including 11 miRNAs (e.g., miR-34b-5p, miR-19b-3p, miR-324-5p, miR-320-3p and miR-322-5p) known to be involved in brain development and the pathogenesis of NDDs. This indicated that dysregulated expression of miRNAs may be involved in the mechanism of glyphosate-induced neurotoxicity. Previous research suggests that miR-34b-5p mediates hippocampal astrocyte apoptosis (39) and also affects target genes, including Numbl and Notch1, which are involved in the Notch signaling pathway (40). The 3'-untranslated regions of $\beta$-catenin and Lef- 1 , which are involved in the Wnt signaling pathway, contain miR-34 binding sites and are sensitive to miR-34b-dependent regulation (41). In addition, the Tcf/Lef transcription factor was identified to be closely associated with the functionality of the miR-34 family (42). The Myc/FOXO3a/mir-34b feedback inhibition loop has also been demonstrated to be involved in regulating cellular proliferation in mammals (43). Furthermore, miR-324-5p is reportedto act as a control in neuronal progenitor cells (44) and contribute to the shift from self-renewal to neuronal differentiation (45).

The present study also identified several potential miRNA target genes and their possible neurological pathways using GO term enrichment and KEGG pathway analysis. The involvement of predicted targets of miRNAs in these biological processes was also determined. The majority of the predicted genes were enriched in neurogenesis regulation, neuron differentiation and brain development, indicating that these targets 


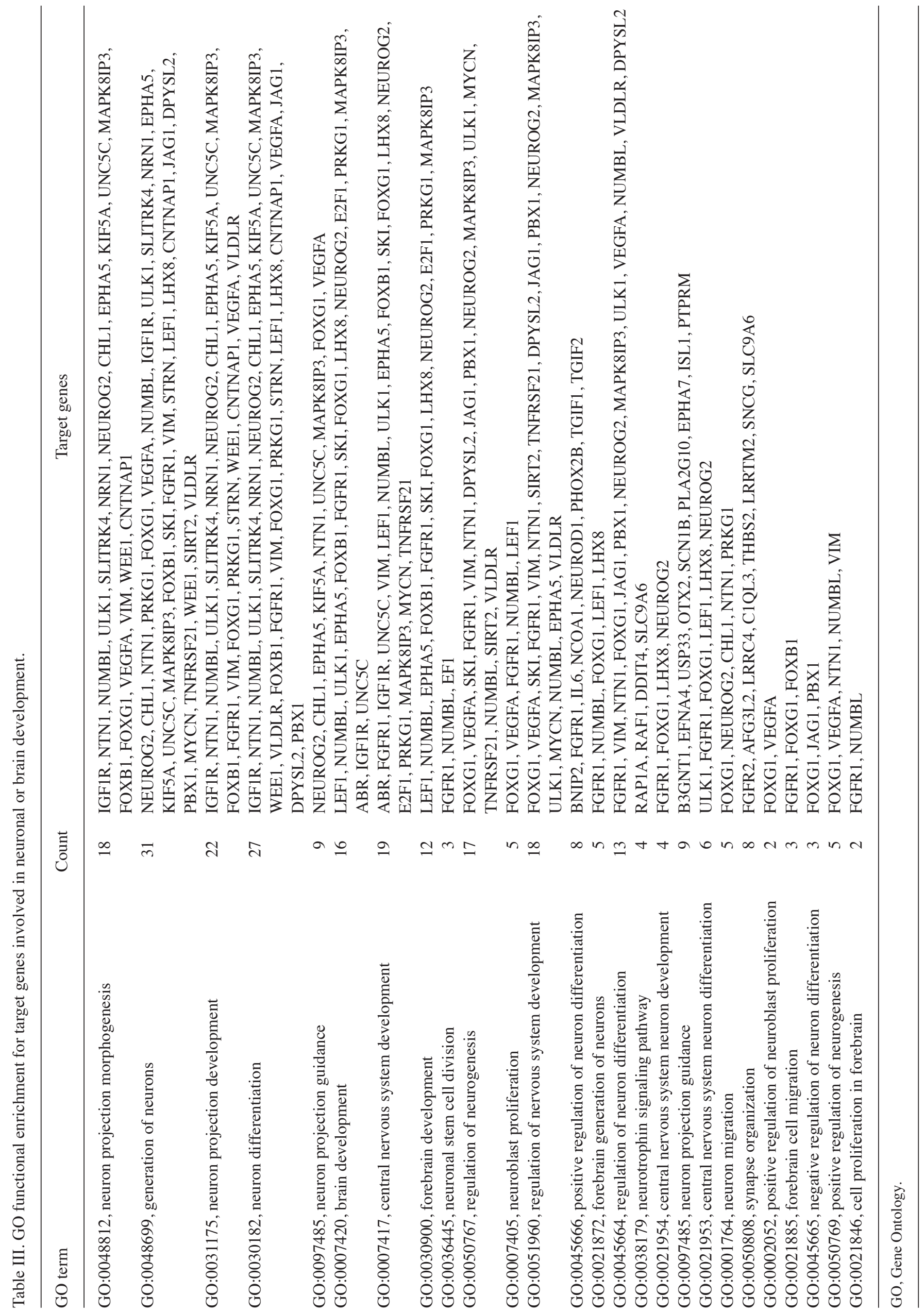


Table IV. KEGG pathway annotations for miRNA targets.

\begin{tabular}{|c|c|c|c|c|}
\hline Pathway ID & Definition & P-value & Count & Genes \\
\hline 4014 & Ras signaling pathway & $1.03134 \times 10^{-6}$ & 20 & $\begin{array}{l}\text { EFNA4, FASL, FGF15, FGFR1, FGFR2, GNG5, } \\
\text { IGF1R, INSR, MAP2K1, PAK4, PAK6, PLA2G10, } \\
\text { PLA2G3, RAF1, RALA, RAP1A, RASSF1, SHOC2, } \\
\text { SOS2, TBK1 }\end{array}$ \\
\hline 4010 & MAPK signaling pathway & 0.00018 & 17 & $\begin{array}{l}\text { CASP3, FASL, FGF15, FGFR1, FGFR2, FLNC, FOS, } \\
\text { GNA12, IL1A, MAP2K1, MAP3K1, MAP4K3, } \\
\text { MKNK1, RAF1, RAP1A, SOS2, TGFBR1 }\end{array}$ \\
\hline 4110 & Cell cycle & 0.00055 & 8 & $\begin{array}{l}\text { CDKN1A, E2F1, E2F5, MCM3, RBL1, RBL2, } \\
\text { WEE1, YWHAQ }\end{array}$ \\
\hline 4068 & FoxO signaling pathway & 0.00087 & 8 & $\begin{array}{l}\text { CCNG2, CDKN1A, FOXG1, IGF1R, PIK3CA, } \\
\text { PLK3, RBL2, SLC2A4 }\end{array}$ \\
\hline 4722 & Neurotrophin signaling pathway & 0.00337 & 9 & $\begin{array}{l}\text { ARHGDIA, FASL, MAP2K1, MAP3K1, NFKBIA, } \\
\text { PRKCD, RAF1, RAP1A, SOS2 }\end{array}$ \\
\hline 4151 & PI3K-Akt signaling pathway & 0.01463 & 11 & $\begin{array}{l}\text { CDKN1A, CRTC2, FGFR1, GNB5, IGF1R, } \\
\text { LAMB3, OSM, PIK3CA, RBL2, VEGFA, YWHAQ }\end{array}$ \\
\hline 4360 & Axon guidance & 0.01524 & 8 & $\begin{array}{l}\text { EFNA4, EPHA7, LIMK2, LRRC4, PAK4, PAK6, } \\
\text { SEMA4C, SEMA6D }\end{array}$ \\
\hline 5214 & Glioma & 0.01612 & 4 & CDKN1A, E2F1, IGF1R, PIK3CA \\
\hline 4730 & Long-term depression & 0.01771 & 5 & GNA12, IGF1R, MAP2K1, PPP2R1A, RAF1 \\
\hline 4066 & HIF-1 signaling pathway & 0.02363 & 5 & CDKN1A, IGF1R, LDHA, PIK3CA, VEGFA \\
\hline 4520 & Adherens junction & 0.02465 & 4 & FGFR1, IGF1R, LEF1, SSX2IP \\
\hline 4024 & cAMP signaling pathway & 0.02635 & 10 & $\begin{array}{l}\text { ADRB2 FOS HCN2 LIPE MAP2K1 NFKBIA } \\
\text { PDE3A PPARA RAF1 RAP1A }\end{array}$ \\
\hline
\end{tabular}

KEGG, Kyoto Encyclopedia of Genes and Genomes; MAPK, mitogen activated protein kinase; PI3K, phosphoinositide-3-kinase; HIF, hypoxia inducible factor; cAMP, cyclic adenosine monophosphate.

may be important in the mechanism of glyphosate-induced neurotoxicity.

It is widely acknowledged that miRNAs can regulate target mRNAs and affect the activities of cells and tissues, implicating the importance of target gene prediction (46). For example, among miR-34b-5p target genes, Chll, Fgfrl, Foxgl and Nrnl were reported to be involved in certain neurological disorders, including autism, schizophrenia and bipolar disorder (47-49).

A large number of the target genes identified in the present study are known to be involved in multiple pathways, including the Wnt and Notch signaling pathways. Certain target genes, including $\mathrm{Numbl}$ and Adam 10, were detected by bioinformatics methods. It is well established that the expression of Numbl and Adam10 in the brain could regulate neural development, synaptogenesis and neural stem cells via the Notch and Wnt/ $\beta$-catenin signaling pathways (50-52). These two pathways are important for maintaining and protecting neural connections in the developing brain $(53,54)$.

In the current study, a PCR array was used to validate the effects of the Wnt and Notch signaling pathways in this study and to help elucidate the mechanism of glyphosate-induced neurotoxicity. A number of targets were identified within the Notch and Wnt signaling pathways that are known to be closely associated with neurogenesis and behavioral deficits in mice. The results of the PCR array indicated that several genes were affected by glyphosate exposure. $\mathrm{Nr} 4 \mathrm{a} 2$ is a transcription factor that is highly expressed in the brain and is crucial for the formation or maintenance of dopaminergic neurons in the central nervous system (CNS) (55). Wnt7b, known to be involved in Wnt signaling, may affect early neural progenitor differentiation by regulating the expression of pro-neural transcription factors, including the T-domain transcription factors Tbr1 and Tbr2 (56). Wnt7b is also involved in Celsr3-Fzd3 signaling, in which it regulates the timing of neural progenitor cell fate via Notch activation (57). In the present study, the miRNA expression levels of $N r 4 a 2$ and $W n t 7 b$ were decreased in the PFC of mouse offspring following glyphosate exposure. These gene expression changes may be associated with abnormal neural differentiation.

$D k k 1$, a secreted inhibitor of $\mathrm{Wnt} / \beta$-catenin signaling, is required for proper neural development and induces the rapid disassembly of synapses (58). Its dysfunction contributes to synaptic degeneration during early stages of neurodegenerative diseases (58), including Alzheimer's disease, Parkinson's disease (PD) and epilepsy (59), as well as impaired motor behavior (60). However, the role of $D k k 1$ in glyphosate-induced neurotoxicity in mouse offspring is completely unknown. Dixdcl, known as a positive regulator of the Wnt signaling pathway, was recently reported to play a role in neurogenesis (61) and the development of cortical dendrites and synapses (62). It is also essential for neural progenitor proliferation and migration during embryonic 


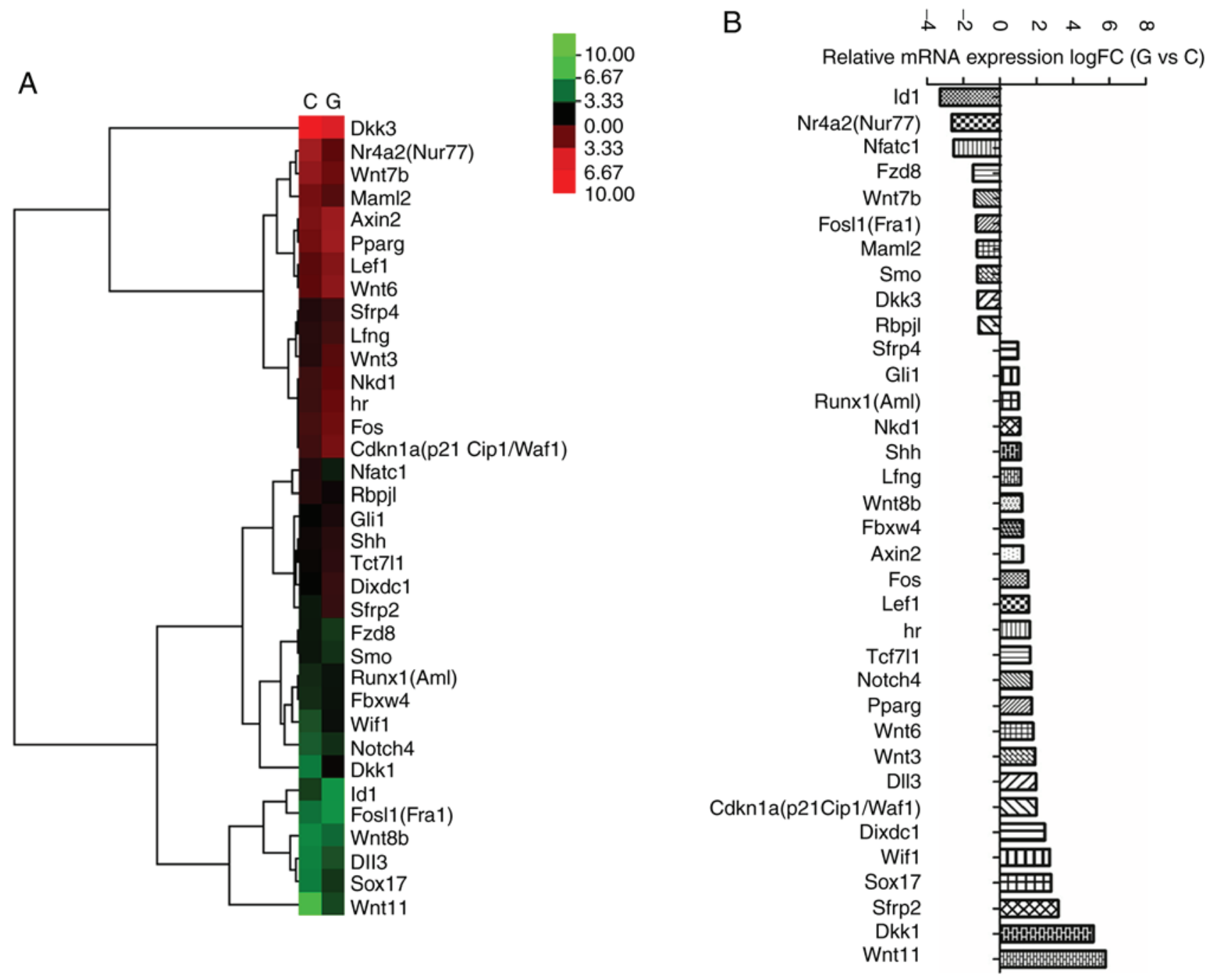

Figure 3. Differentially expressed genes involved in the Wnt and Notch pathways. The mouse Wnt and Notch signaling pathway RT2 profiler ${ }^{\mathrm{TM}}$ PCR array plates were used, which contained 84 key genes involved in the Wnt pathway and 26 key genes involved in the Notch pathway. (A) Each column represents a sample; each row represents a gene. Red color indicates upregulated genes; green color indicates downregulated genes in the glyphosate group. Genes were considered differentially expressed at fold change $\leq 0.5$ and $\geq 2$. (B) Relative mRNA expression in the two groups. PCR, polymerase chain reaction; C, control group; G, glyphosate-treated group.

cortical development (63). Rare missense variants in Dixdcl have been identified in ASD patient cohorts via genetic sequencing (63), indicating that Dixdcl may be associated with morphological defects associated with NDDs. Runxl and $S h h$, which are involved in the Notch signaling pathway, have been reported to serve critical functions in the developing brain (64-66). For example, Runxl may be upregulated after injury to promote neuronal differentiation, in order to facilitate repair of the CNS. Therefore, upregulated Runx expression is associated with brain injury and disease (64). Shh influences neurogenesis and neural patterning during development of the CNS (67). Dysregulated Shh signaling may lead to neurological disorders such as ASD, depression and PD, as well as locomotor deficits (68). Lef- 1 , a direct Wnt/ $\beta$-catenin signaling target (69), is a crucial determinant of neurogenesis and of neural progenitor fates in the brain $(70,71)$. It also regulates $\beta$-catenin-dependent transcription of neural progenitor genes in the neocortex (72). Axin2, a classic Wnt target gene and $\beta$-catenin destruction complex scaffolding protein, has been reported to control the switch of intermediate progenitors from a proliferative to a differentiated status in the developing cerebral cortex (73). Enhancing Axin expression in neuronal progenitors leads to an enlarged neocortex and autistic-like behaviors (74). In the present study, the mRNA expression levels of Dkk1, Dixdc1, Runx1, Shh, Lef-1 and Axin2 were elevated in the PFC of mouse offspring following glyphosate exposure. These gene expression changes may be associated with neural behavior abnormalities (including anxiety- and depression-like behaviors and decreased social interaction behaviors; data not shown) observed in the mouse offspring. Therefore, these genes maybe good targets for the prevention of glyphosate-induced neurotoxicity in the developing brain.

The present study was limited by utilizing a single treatment dose to examine miRNA expression changes in mouse offspring caused by glyphosate exposure during pregnancy and lactation, and the simple miRNA prediction using bioinformatics was not sufficient. Future studies will utilize multiple treatment doses to better illustrate the results and verify the authenticity and accuracy of the predicted target genes by luciferase gene assay. These additional measures will increase 
our understanding of the mechanism of glyphosate-induced neurotoxicity and will help clarify the association between glyphosate and NDDs.

In conclusion, the current study focused on the changes in miRNA expression in the PFC of mouse offspring that were exposed to glyphosate during pregnancy and lactation. An miRNA microarray and PCR array were performed to examine the effects of glyphosate on the brain. The current findings provide a basis for identifying the mechanism of action of glyphosate-induced neurotoxicity in the developing brain, and for clarifying the association between glyphosate and NDDs.

\section{Acknowledgements}

The present study was supported by the Natural Science Foundation of Zhejiang Province of China (grant no. LY17H090001) and the Medical Scientific Research Foundation of Zhejiang Province of China (grant no. 2013KYA049).

\section{References}

1. Hernández-Plata I, Giordano $M$, Díaz-Muñoz $M$ and Rodriguez VM: The herbicide glyphosate causes behavioral changes and alterations in dopaminergic markers in male Sprague-Dawley rat. Neurotoxicology 46: 79-91, 2015.

2. Paganelli A, Gnazzo V, Acosta H, López SL and Carrasco AE: Glyphosate-based herbicides produce teratogenic effects on vertebrates by impairing retinoic acid signaling. Chem Res Toxicol 23: 1586-1595, 2010.

3. Guyton KZ, Loomis D, Grosse Y, El Ghissassi F, Benbrahim-Tallaa L, Guha N, Scoccianti C, Mattock H and Straif K; International Agency for Research on Cancer Monograph Working Group, IARC, Lyon, France: Carcinogenicity of tetrachlorvinphos, parathion, malathion, diazinon, and glyphosate. Lancet Oncol 16: 490-491, 2015.

4. Williams GM, Kroes R and Munro IC: Safety evaluation and risk assessment of the herbicide Roundup and its active ingredient, glyphosate, for humans. Regul Toxicol Pharmacol 31: 117-165, 2000 .

5. Gallegos CE, Bartos M, Bras C, Gumilar F, Antonelli MC and Minetti A: Exposure to a glyphosate-based herbicide during pregnancy and lactation induces neurobehavioral alterations in rat offspring. Neurotoxicology 53: 20-28, 2016.

6. Sobjak TM, Romão S, do Nascimento CZ, Dos Santos AFP, Vogel L and Guimarães ATB: Assessment of the oxidative and neurotoxic effects of glyphosate pesticide on the larvae of Rhamdia quelen fish. Chemosphere 182: 267-275, 2017.

7. de Araujo JS, Delgado IF and Paumgartten FJ: Glyphosate and adverse pregnancy outcomes, a systematic review of observational studies. BMC Public Health 16: 472, 2016.

8. Poulsen MS, Rytting E, Mose T and Knudsen LE: Modeling placental transport: Correlation of in vitro BeWo cell permeability and ex vivo human placental perfusion. Toxicol In Vitro 23: 1380-1386, 2009.

9. Coullery RP, Ferrari ME and Rosso SB: Neuronal development and axon growth are altered by glyphosate through a WNT non-canonical signaling pathway. Neurotoxicology 52: 150-161, 2016.

10. Shelton JF, Geraghty EM, Tancredi DJ, Delwiche LD, Schmidt RJ, Ritz B, Hansen RL and Hertz-Picciotto I: Neurodevelopmental disorders and prenatal residential proximity to agricultural pesticides: The CHARGE study. Environ Health Perspect 122: 1103-1109, 2014.

11. Rangasamy S, D'Mello SR and Narayanan V: Epigenetics, autism spectrum, and neurodevelopmental disorders. Neurotherapeutics 10: 742-756, 2013.

12. Xu Y, Chen XT, Luo M, Tang Y, Zhang G, Wu D, Yang B, Ruan DY and Wang HL: Multiple epigenetic factors predict the attention deficit/hyperactivity disorder among the Chinese Han children. J Psychiatr Res 64: 40-50, 2015.
13. Bartel DP: MicroRNAs: Genomics, biogenesis, mechanism, and function. Cell 116: 281-297, 2004.

14. Witwer KW, Sisk JM, Gama L and Clements JE: MicroRNA regulation of IFN-beta protein expression: Rapid and sensitive modulation of the innate immune response. J Immunol 184: 2369-2376, 2010

15. Wu L, Li H, Jia CY, Cheng W, Yu M, Peng M, Zhu Y, Zhao Q, Dong YW, Shao K, et al: MicroRNA-223 regulates FOXO1 expression and cell proliferation. FEBS Lett 586: 1038-1043, 2012.

16. Kandemir H, Erdal ME, Selek S, Ay Öİ, Karababa IF, Kandemir SB, Ay ME, Yılmaz ŞG, Bayazıt H and Taşdelen B: Evaluation of several micro RNA (miRNA) levels in children and adolescents with attention deficit hyperactivity disorder. Neurosci Lett 580: 158-162, 2014.

17. Casey BJ, Epstein JN, Buhle J, Liston C, Davidson MC Tonev ST, Spicer J, Niogi S, Millner AJ, Reiss A, et al: Frontostriatal connectivity and its role in cognitive control in parent-child dyads with ADHD. Am J Psychiatry 164: 1729-1736, 2007.

18. Somel M, Liu X, Tang L, Yan Z, Hu H, Guo S, Jiang X, Zhang X, $\mathrm{Xu} \mathrm{G}, \mathrm{Xie} \mathrm{G}$, et al: MicroRNA-driven developmental remodeling in the brain distinguishes humans from other primates. PLoS Biol 9: e1001214, 2011.

19. Wu LH, Peng M, Yu M, Zhao QL, Li C, Jin YT, Jiang Y, Chen ZY, Deng NH, Sun H and Wu XZ: Circulating MicroRNA Let-7d in attention-deficit/hyperactivity disorder. Neuromolecular Med 17: 137-146, 2015.

20. Wu L, Zhao Q, Zhu X, Peng M, Jia C, Wu W, Zheng J and Wu XZ: A novel function of microRNA let-7d in regulation of galectin-3 expression in attention deficit hyperactivity disorder rat brain. Brain Pathol 20: 1042-1054, 2010.

21. Hollins SL, Goldie BJ, Carroll AP, Mason EA, Walker FR, Eyles DW and Cairns MJ: Ontogeny of small RNA in the regulation of mammalian brain development. BMC Genomics 15: 777 , 2014.

22. Shioya M, Obayashi S, Tabunoki H, Arima K, Saito Y, Ishida T and Satoh J: Aberrant microRNA expression in the brains of neurodegenerative diseases: miR-29a decreased in Alzheimer disease brains targets neurone navigator 3. Neuropathol Appl Neurobiol 36: 320-330, 2010.

23. Zhu JJ, Liu YF, Zhang YP, Zhao CR, Yao WJ, Li YS, Wang KC, Huang TS, Pang W, Wang XF, et al: VAMP3 and SNAP23 mediate the disturbed flow-induced endothelial microRNA secretion and smooth muscle hyperplasia. Proc Natl Acad Sci USA 114: 8271-8276, 2017.

24. Livak KJ and Schmittgen TD: Analysis of relative gene expression data using real-time quantitative PCR and the 2(-Delta Delta C(T)) method. Methods 25: 402-408, 2001.

25. Alsharafi WA, Xiao B and Li J: MicroRNA-139-5p negatively regulates NR2A-containing NMDA receptor in the rat pilocarpine model and patients with temporal lobe epilepsy. Epilepsia 57: 1931-1940, 2016.

26. Srivastav S, Walitza $S$ and Grünblatt E: Emerging role of miRNA in attention deficit hyperactivity disorder: A systematic review. Atten Defic Hyperact Disord, May 10, 2017 (Epub ahead of print).

27. Luo SX and Huang EJ: Dopaminergic neurons and brain reward pathways: From neurogenesis to circuit assembly. Am J Pathol 186: 478-488, 2016.

28. Trujillo-Paredes N, Valencia C, Guerrero-Flores G, Arzate DM, Baizabal JM, Guerra-Crespo M, Fuentes-Hernández A, Zea-Armenta I and Covarrubias L: Regulation of differentiation flux by Notch signalling influences the number of dopaminergic neurons in the adult brain. Biol Open 5: 336-347, 2016.

29. Cattani D, de Liz Oliveira Cavalli VL, Heinz Rieg CE, Domingues JT, Dal-Cim T, Tasca CI, Mena Barreto Silva FR and Zamoner A: Mechanisms underlying the neurotoxicity induced by glyphosate-based herbicide in immature rat hippocampus: Involvement of glutamate excitotoxicity. Toxicology 320: 34-45, 2014.

30. Yu CJ, Du JC, Chiou HC, Chung MY, Yang W, Chen YS, Fuh MR, Chien LC, Hwang B and Chen ML: Increased risk of attention-deficit/hyperactivity disorder associated with exposure to organophosphate pesticide in Taiwanese children. Andrology 4: 695-705, 2016.

31. Marks AR, Harley K, Bradman A, Kogut K, Barr DB, Johnson C, Calderon $\mathrm{N}$ and Eskenazi B: Organophosphate pesticide exposure and attention in young Mexican-American children: The CHAMACOS study. Environ Health Perspect 118: 1768-1774, 2010. 
32. Eubig PA, Aguiar A and Schantz SL: Lead and PCBs as risk factors for attention deficit/hyperactivity disorder. Environ Health Perspect 118: 1654-1667, 2010.

33. Mill $\mathrm{J}$ and Petronis A: Pre- and peri-natal environmental risks for attention-deficit hyperactivity disorder (ADHD): The potential role of epigenetic processes in mediating susceptibility. J Child Psychol Psychiatry 49: 1020-1030, 2008.

34. Callaway E: Epigenomics starts to make its mark. Nature 508: $22,2014$.

35. Murgatroyd C, Patchev AV, Wu Y, Micale V, Bockmühl Y, Fischer D, Holsboer F, Wotjak CT, Almeida OF and Spengler D: Dynamic DNA methylation programs persistent adverse effects of early-life stress. Nat Neurosci 12: 1559-1566, 2009.

36. $\mathrm{Hu} \mathrm{Z}$ and Li Z: miRNAs in synapse development and synaptic plasticity. Curr Opin Neurobiol 45: 24-31, 2017.

37. Huntzinger $\mathrm{E}$ and Izaurralde $\mathrm{E}$ : Gene silencing by microRNAs: Contributions of translational repression and mRNA decay. Nat Rev Genet 12: 99-110, 2011.

38. Fabian MR, Sonenberg N and Filipowicz W: Regulation of mRNA translation and stability by microRNAs. Annu Rev Biochem 79: 351-379, 2010.

39. Liu L, Liu L, Shi J, Tan M, Xiong J, Li X, Hu Q, Yi Z and Mao D: MicroRNA-34b mediates hippocampal astrocyte apoptosis in a rat model of recurrent seizures. BMC Neurosci 17: 56, 2016.

40. Luceri C, Bigagli E, Pitozzi V and Giovannelli L: A nutrigenomics approach for the study of anti-aging interventions: Olive oil phenols and the modulation of gene and microRNA expression profiles in mouse brain. Eur J Nutr 56: 865-877, 2017.

41. Kim NH, Kim HS, Li XY, Lee I, Choi HS, Kang SE, Cha SY, Ryu JK, Yoon D, Fearon ER, et al: A p53/miRNA-34 axis regulates Snail1-dependent cancer cell epithelial-mesenchymal transition. J Cell Biol 195: 417-433, 2011.

42. Cha YH, Kim NH, Park C, Lee I, Kim HS and Yook JI: miRNA-34 intrinsically links p53 tumor suppressor and Wnt signaling. Cel Cycle 11: 1273-1281, 2012

43. Isik M, Blackwell TK and Berezikov E: MicroRNA mir-34 provides robustness to environmental stress response via the DAF-16 network in C. elegans. Sci Rep 6: 36766, 2016.

44. Ferretti E, De Smaele E, Miele E, Laneve P, Po A, Pelloni M, Paganelli A, Di Marcotullio L, Caffarelli E, Screpanti I, et al: Concerted microRNA control of Hedgehog signalling in cerebellar neuronal progenitor and tumour cells. EMBO J 27: 2616-2627, 2008

45. Stappert L, Borghese L, Roese-Koerner B, Weinhold S, Koch P, Terstegge S, Uhrberg M, Wernet $\mathrm{P}$ and Brüstle $\mathrm{O}$ MicroRNA-based promotion of human neuronal differentiation and subtype specification. PloS one 8: e59011, 2013

46. Andrés-León E, Gómez-López G and Pisano DG: Prediction of miRNA-mRNA interactions using miRGate. Methods Mol Biol 1580: 225-237, 2017.

47. Salyakina D, Cukier HN, Lee JM, Sacharow S, Nations LD, Ma D, Jaworski JM, Konidari I, Whitehead PL, Wright HH, et al Copy number variants in extended autism spectrum disorder families reveal candidates potentially involved in autism risk. PLoS One 6: e26049, 2011.

48. Pedrosa E, Shah A, Tenore C, Capogna M, Villa C, Guo X, Zheng D and Lachman HM: $\beta$-catenin promoter ChIP-chip reveals potential schizophrenia and bipolar disorder gene network. J Neurogenet 24: 182-193, 2010.

49. Fimiani C, Goina E, Su Q, Gao G and Mallamaci A: RNA activation of haploinsufficient Foxg1 gene in murine neocortex. Sci Rep 6: 39311, 2016.

50. Ebbing EA,Medema JP,Damhofer H,Meijer SL,Krishnadath KK, van Berge Henegouwen MI, Bijlsma MF and van Laarhoven HW: ADAM10-mediated release of heregulin confers resistance to trastuzumab by activating HER3. Oncotarget 7: 10243-10254, 2016.

51. Saftig P and Lichtenthaler SF: The alpha secretase ADAM10: A metalloprotease with multiple functions in the brain. Prog Neurobiol 135: 1-20, 2015.

52. Nishimura T and Kaibuchi K: Numb controls integrin endocytosis for directional cell migration with aPKC and PAR-3. Dev Cell 13: 15-28, 2007.

53. Price DJ, Kennedy H, Dehay C, Zhou L, Mercier M, Jossin Y, Goffinet AM, Tissir F, Blakey D and Molnár Z: The development of cortical connections. Eur J Neurosci 23: 910-920, 2006.

54. Bonini SA, Ferrari-Toninelli G, Maccarinelli G, Bettinsoli P, Montinaro $\mathrm{M}$ and Memo M: Cytoskeletal protection: Acting on notch to prevent neuronal dysfunction. Neurodegener Dis 13: 93-95, 2014.
55. Goodings L, He J, Wood AJ, Harris WA, Currie PD and Jusuf PR In vivo expression of Nurr1/Nr4a2a in developing retinal amacrine subtypes in zebrafish $\mathrm{Tg}(\mathrm{nr} 4 \mathrm{a} 2 \mathrm{a}: \mathrm{eGFP})$ transgenics. J Comp Neurol 525: 1962-1979, 2017.

56. Papachristou P, Dyberg C, Lindqvist M, Horn Z and Ringstedt $T$ : Transgenic increase of Wnt7b in neural progenitor cells decreases expression of T-domain transcription factors and impairs neuronal differentiation. Brain Res 1576: 27-34, 2014.

57. Wang W, Jossin Y, Chai G, Lien WH, Tissir F and Goffinet AM: Feedback regulation of apical progenitor fate by immature neurons through Wnt7-Celsr3-Fzd3 signalling. Nat Commun 7: 10936, 2016.

58. Dickins EM and Salinas PC: Wnts in action: From synapse formation to synaptic maintenance. Front Cell Neurosci 7: 162, 2013.

59. Scott EL and Brann DW: Estrogen regulation of Dkk1 and Wnt $/ \beta$-Catenin signaling in neurodegenerative disease. Brain Res 1514: 63-74, 2013

60. Galli S, Lopes DM, Ammari R, Kopra J, Millar SE, Gibb A and Salinas PC: Deficient Wnt signalling triggers striatal synaptic degeneration and impaired motor behaviour in adult mice. Nat Commun 5: 4992, 2014

61. Lu H, Jiang R, Tao X, Duan C, Huang J, Huan W, He Y, Ge J and Ren J: Expression of Dixdc1 and its role in astrocyte proliferation after traumatic brain injury. Cell Mol Neurobiol 37: 1131-1139, 2017.

62. Kwan V, Meka DP, White SH, Hung CL, Holzapfel NT, Walker S, Murtaza N, Unda BK, Schwanke B, Yuen RKC, et al: DIXDC1 Phosphorylation and control of dendritic morphology are impaired by rare genetic variants. Cell Rep 17: 1892-1904, 2016.

63. Singh KK, Ge X, Mao Y, Drane L, Meletis K, Samuels BA and Tsai LH: Dixdc1 is a critical regulator of DISC1 and embryonic cortical development. Neuron 67: 33-48, 2010.

64. Wang JW and Stifani S: Roles of Runx genes in nervous system development. Adv Exp Med Biol 962: 103-116, 2017.

65. Zagami CJ and Stifani S: Molecular characterization of the mouse superior lateral parabrachial nucleus through expression of the transcription factor Runx1. PLoS One 5: e13944, 2010.

66. Muthu V, Eachus H, Ellis P, Brown S and Placzek M: Rx3 and Shh direct anisotropic growth and specification in the zebrafish tuberal/anterior hypothalamus. Development 143: 2651-2663, 2016.

67. Feijóo CG, Oñate MG, Milla LA and Palma VA: Sonic hedgehog (Shh)-Gli signaling controls neural progenitor cell division in the developing tectum in zebrafish. Eur J Neurosci 33: 589-598, 2011.

68. Patel SS, Tomar S, Sharma D, Mahindroo N and Udayabanu M Targeting sonic hedgehog signaling in neurological disorders. Neurosci Biobehav Rev 74: 76-97, 2017.

69. Zhang J, Gotz S, Vogt Weisenhorn DM, Simeone A, Wurst W and Prakash N: A WNT1-regulated developmental gene cascade prevents dopaminergic neurodegeneration in adult En1(+/-) mice. Neurobiol Dis 82: 32-45, 2015.

70. Galceran J, Miyashita-Lin EM, Devaney E, Rubenstein JL and Grosschedl R: Hippocampus development and generation of dentate gyrus granule cells is regulated by LEF1. Development 127: 469-482, 2000.

71. Ki H, Jung HC, Park JH, Kim JS, Lee KY, Kim TS and Kim K: Overexpressed LEF-1 proteins display different nuclear localization patterns of beta-catenin in normal versus tumor cells. Cell Biol Int 30: 253-261, 2006.

72. Kuwahara A, Sakai H, Xu Y, Itoh Y, Hirabayashi Y and Gotoh Y: Tcf3 represses Wnt- $\beta$-catenin signaling and maintains neural stem cell population during neocortical development. PLoS One 9: e94408, 2014.

73. Mutch CA, Schulte JD, Olson E and Chenn A: Beta-catenin signaling negatively regulates intermediate progenitor population numbers in the developing cortex. PLoS One 5: e12376, 2010

74. Pérez-Palma E, Andrade V, Caracci MO, Bustos BI, Villaman C, Medina MA, Ávila ME, Ugarte GD and De Ferrari GV: Early transcriptional changes induced by $\mathrm{Wnt} / \beta$-catenin signaling in hippocampal neurons. Neural Plast 2016: 4672841, 2016.

This work is licensed under a Creative Commons Attribution-NonCommercial-NoDerivatives 4.0 International (CC BY-NC-ND 4.0) License. 